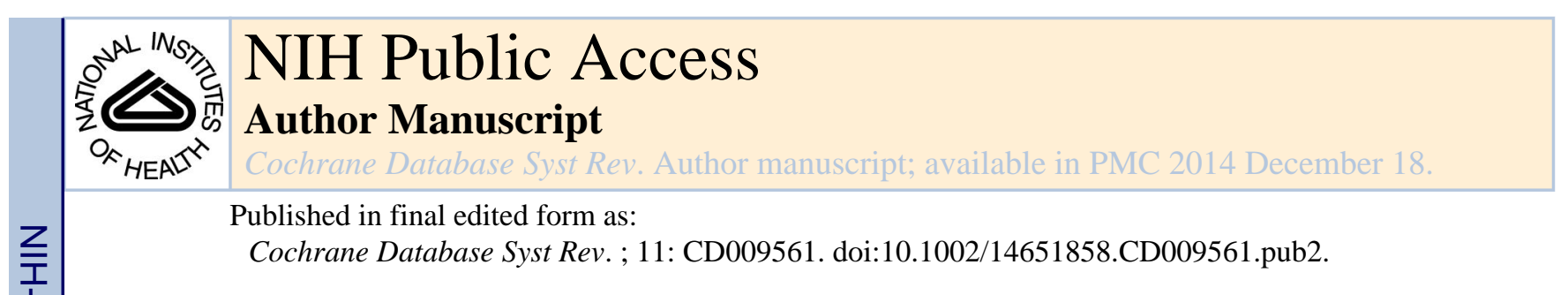

\title{
Artificial corneas versus donor corneas for repeat corneal transplants
}

\author{
Esen K Akpek ${ }^{1}$, Majed Alkharashi ${ }^{2}$, Frank S Hwang ${ }^{3}$, Sueko $\mathbf{M ~ N g}^{4}$, and Kristina Lindsley ${ }^{4}$ \\ ${ }^{1}$ Wilmer Eye Institute, Johns Hopkins University School of Medicine, Baltimore, MD, USA \\ ${ }^{2}$ Department of Ophthalmology, King Saud University, Riyadh, Saudi Arabia \\ ${ }^{3}$ Cornea, External Disease and Refractive Surgery, Kresge Eye Institute, Detroit, Michigan, USA \\ ${ }^{4}$ Department of Epidemiology, Johns Hopkins Bloomberg School of Public Health, Baltimore, \\ Maryland, USA
}

\begin{abstract}
Background-Individuals who have failed one or more full thickness penetrating keratoplasties (PKs) may be offered repeat corneal surgery using an artificial or donor cornea. An artificial or prosthetic cornea is known as a keratoprosthesis. Both donor and artificial corneal transplantations involve removal of the diseased and opaque recipient cornea (or the previously failed cornea) and replacement with another donor or prosthetic cornea.
\end{abstract}

Objectives-To assess the effectiveness of artificial versus donor corneas in individuals who have had one or more failed donor corneal transplantations.

\footnotetext{
Copyright @ 2014 The Cochrane Collaboration. Published by John Wiley \& Sons, Ltd. Maumenee \#317, Baltimore, MD, 21287, USA. esakpek@jhmi.edu.

CONTRIBUTIONS OF AUTHORS

Conceiving and designing the review: EKA

Coordinating the review: KL

Data collection for the review

Designing electronic search strategies: Iris Gordon at the CEVG editorial base

Undertaking manual searches: EKA, KL

Screening search results: MA, FSH, SMN, KL

Organising retrieval of papers: SMN, KL

Screening retrieved papers against inclusion criteria: MA, FSH, SMN, KL

Appraising quality of papers: MA, FSH, SMN, KL

Extracting data from papers: MA, FSH, SMN, KL

Data management for the review: KL, SMN

Entering data into RevMan: KL, SMN

Interpretation of data

Providing a methodological perspective: KL, SMN

Providing a clinical perspective: EKA, MA, FSH

Providing a policy perspective: EKA, MA, FSH

Providing a consumer perspective: EKA, MA, FSH

Writing the review: EKA, KL, MA, FSH, SMN

Providing general advice on the review: EKA, KL, MA, FSH, SMN

Performing previous work that was the foundation of the current study: EKA

DECLARATIONS OF INTEREST

None known.
}

Contact address: Esen K Akpek, Wilmer Eye Institute, Johns Hopkins University School of Medicine, 600 N. Wolfe Street, 
Search methods-We searched CENTRAL (which contains the Cochrane Eyes and Vision Group Trials Register) (2013, Issue 10), Ovid MEDLINE, Ovid MEDLINE In-Process and Other Non-Indexed Citations, Ovid MEDLINE Daily, Ovid OLDMEDLINE (January 1946 to November 2013), EMBASE (January 1980 to November 2013), Latin American and Caribbean Health Sciences Literature Database (LILACS) (January 1982 to November 2013), the metaRegister of Controlled Trials ( $m \mathrm{RCT}$ ) (www.controlled-trials.com), ClinicalTrials.gov (www.clinicaltrials.gov) and the World Health Organization (WHO) International Clinical Trials Registry Platform (ICTRP) (www.who.int/ictrp/search/en). We did not use any date or language restrictions in the electronic searches for trials. We last searched the electronic databases on 27 November 2013.

Selection criteria-Two review authors independently assessed reports from the electronic searches to identify randomized controlled trials (RCTs) or controlled clinical trials (CCTs). We resolved discrepancies by discussion or consultation with a third review author.

Data collection and analysis-For discussion purposes, we assessed findings from observational cohort studies and non-comparative case series. No data synthesis was performed.

Main results-We did not identify any RCTs or CCTs comparing artificial corneas with donor corneas for repeat corneal transplantations.

Authors' conclusions-The optimal management for those individuals who have failed a conventional corneal transplantation is not known. Currently, in some centers, artificial corneal devices routinely are recommended after just one graft failure, and in others, not until after multiple graft failures, or not at all. To date, there have been no controlled trials comparing the visual outcomes and complications of artificial corneal devices (particularly the Boston type 1 keratoprosthesis which is the most commonly implanted artificial corneal device) with repeat donor corneal transplantation, in order to guide surgeons and their patients. It is apparent that such a trial is needed and would offer significant benefit to an ever-increasing pool of people with visual disability due to corneal opacification, most of whom are still in productive stages of their lives.

\section{PLAIN LANGUAGE SUMMARY}

\section{Artificial corneal devices versus human donor corneas for people undergoing repeat corneal transplantation}

Review question-Our objective was to compare the success of artificial corneal devices versus donor (human) corneas in people who had already had at least one donor cornea transplant that had not worked.

Background-There are several types of corneal transplantations. The type of transplantation depends on how much of the cornea (front part of the eye) is removed and replaced. When the entire cornea is removed and replaced completely, it is called a full thickness penetrating keratoplasty (PK). If only part of the cornea is removed and replaced by the same part from a donor, the surgery is named based on the parts transplanted (e.g. endothelial keratoplasty (EK) refers to transplantation of the inner or endothelial layers of the cornea). 
Corneal transplantation is one of the most successful tissue or organ transplantations.

However, sometimes the transplantation fails and the donor tissue becomes opaque (cloudy). We looked only at people who had failed at least one PK and required another corneal surgery.

In some medical centers, artificial corneas are recommended after a person has had just one failure of a donor PK; in other centers, an artificial cornea is not recommended until after a person has had several donor PKs fail. At some centers an artificial cornea is never offered. Currently, no guidelines are available as to how many donor corneal transplants can be performed in an eye that has failed a corneal transplantation.

Corneal transplantation with either a donor cornea or an artificial cornea (known as a keratoprosthesis) involves removal of the cloudy cornea and replacement with a clear cornea or corneal device. Some examples of keratoprostheses (KPros) are the Boston KPro (Types I and II), AlphaCor, osteo-odonto-keratoprosthesis (OOKP), and Fyodorov-Zuev KPro. The Boston KPro is the most commonly implanted artificial corneal device.

Key results and conclusions-We found no controlled trials that have compared the success, including vision and side effects, of artificial corneas against donor corneas in people whose previous donor corneal transplants have failed. The best treatment for someone whose PK has failed is not known. There is no high-quality evidence to guide surgeons and patients as to the best treatment to use after a donor transplant fails. Therefore, a trial comparing the use of artificial versus human donor corneas after PK failure is needed. Comparative clinical trials are needed to provide the missing information and would offer significant benefit to an ever-increasing pool of people with visual disability due to a cloudy cornea, most of whom are still at working age or in school.

\section{BACKGROUND}

\section{Description of the condition}

The cornea is the transparent layer of the anterior surface of the eye and functions to transmit light and provide most of the refractive power of the eye. Diseases affecting the cornea are a major cause of blindness worldwide. Globally, corneal opacity is the fourth leading cause of bilateral blindness after cataract, glaucoma, and age-related macular degeneration, affecting some four to eight million people, $90 \%$ of whom live in the developing world (Murthy 2012; Whitcher 2001). Furthermore, unilateral corneal blindness, which also creates much disability, is not reflected in these statistics. Additionally, children and young adults are affected by corneal blindness proportionately more than by other major blinding diseases, such as macular degeneration and glaucoma, which are associated with aging.

Unfortunately, there are currently no proven medical treatments available to restore clarity in diseased corneas. Surgery with donor corneal transplantation (keratoplasty) is the definitive treatment for corneal blindness. Although the majority of these transplants are successful, if the transplantation fails the first time for any reason, a repeat transplantation using an artificial or donor cornea may be considered. 


\section{Description of the intervention}

Corneal transplantation is one of the most commonly performed transplant procedures in the developed world. A total of 46,892 corneal transplantations were performed in the United States in 2013 (EBAA 2013). Successful transplantation occurs when the corneal graft is not rejected and it retains clarity and integrity. Several recently published studies documented that the single most important factor affecting the success of corneal transplantation is the pre-operative indication for the surgery (Thompson 2003; Wagoner 2009). Donor corneal transplantation achieves remarkable overall success rates with approximately $90 \%$ of "low risk" patients having successful transplants (ACGR 1993; Thompson 2003; Wagoner 2009). Patients at "low risk" of corneal graft failure typically suffer vision loss from corneal shape problems (such as keratoconus) or from loss of clarity due to genetic problems of the endothelial layer of the cornea (such as Fuchs endothelial dystrophy), trauma or infection. For example, although the five-year survival probability is usually over $90 \%$ for keratoconus, this rate is less than $50 \%$ when the indication is corneal edema due to endothelial failure from intraocular surgery, and even lower when the eye is aphakic (without the natural lens) (ACGR 1993; Thompson 2003).

The likelihood of corneal graft survival drops markedly in the setting of previous corneal graft failure. Re-graft (repeat donor corneal transplantation), presence of significant neovascularization of the host bed, history of glaucoma and previous herpetic infection also decrease the likelihood of graft survival (ACGR 1993; Siganos 2010; Thompson 2003; Wagoner 2009). Overall risk of failure for re-grafts is about $50 \%$ at five years (Thompson 2003; Yildiz 2010), although this percentage is probably an underestimate as most surgeons tend to limit re-grafting only to patients in whom they believe there is a reasonable chance of success for subsequent graft survival and improvement in visual acuity. Of note, about $10 \%$ to $16 \%$ of all corneal transplants around the world are re-grafts (EBAA 2013; Thompson 2003; Yildiz 2010).

In the United States, patients who have failed multiple corneal grafts may be offered an artificial cornea. An artificial or prosthetic cornea, known as a keratoprosthesis (KPro), is a corneal implant made of synthetic material, the most common of which is the Boston keratoprosthesis (Boston KPro). The Boston KPro is a two piece, collar-button device made of polymethylmethacrylate (PMMA), a transparent thermoplastic, with a titanium locking ring. Although it is associated with some complications, such as infection, extrusion, glaucoma, and retinal detachment, because it is made of artificial material it will not opacify (become cloudy). The AlphaCor device, made from poly-2-hydroxyethyl methacrylate, is made of a one-piece flexible implant with a peripheral skirt and a transparent central region connected on a molecular level by an interpenetrating polymer network. It is implanted in the recipient eye via a two-stage surgical procedure. Osteo-odonto-keratoprosthesis, reserved for individuals with severe ocular surface disorders, requires a complex multi-step surgical procedure and, therefore, is performed very infrequently. A lamina of the patient's tooth is grafted into the eye after having been transplanted elsewhere for biointegration and vascularization purposes. An artificial optic made of PMMA is then installed in the unit to allow vision. The Fyodorov-Zuev keratoprosthesis, commonly used in the former Soviet Union, is made of a titanium supporting plate with two large openings to allow aqueous 
humor to flow anteriorly. An optical cylinder is screwed into the center of the supporting plate, then the assembled device is implanted.

In general, artificial corneal implantation requires long-term topical and sometimes oral treatment to prevent or treat complications such as infections and glaucoma. Close postoperative follow-up is required due to risk of complications, which can sometimes lead to permanent blindness, particularly due to retinal detachment and endophthalmitis. These complication rates have been well monitored by surveillance studies (Boston Type 1 KPro Study; Hicks 2006). The rates of vision-threatening complications and visual outcomes on the other hand are less well studied in repeat donor corneal transplantation cases. However, the rates of corneal melting and infection certainly are expected to be lower with donor transplantation than with artificial devices.

\section{How the intervention might work}

Both donor and artificial corneal transplantations involve removal of the diseased and opaque portion of the recipient cornea, and replacement with clear cornea or corneal device. The surgical procedure using an artificial cornea is similar to full thickness corneal transplantation using a donor cornea (penetrating keratoplasty, PK) once the device has been assembled.

The Boston KPro, the most commonly implanted artificial corneal device, comes in two main types (Aquavella 2005; Ilhan-Sarac 2005; Ma 2005). Type I is the most commonly used style in the United States. It consists of two plastic parts: an anterior part which houses the refractive portion, and a removable perforated back plate. The device requires donor corneal tissue to be sandwiched between the two plates. The holes in the back plate are thought to enhance nutrition and rehydration of the clamped corneal stroma adjacent to the stem, which may help to prevent necrosis of the surrounding tissue. In addition, the device has a titanium locking c-ring to secure the unit after its assembly and prevent unscrewing of the back plate. After assembly, the whole device is then sutured into the recipient eye in the same manner as with donor corneal transplantation. A newer generation type I KPro with a back plate made of titanium recently has been approved by the United States Food and Drug Administration (MEEI 2013). This model does not require a locking c-ring (Todani 2011).

The type I Boston KPro is indicated in eyes with sufficient tear secretion and normal blinking. The longer type II device is similar to type I, except for an additional $2 \mathrm{~mm}$ long anterior nub for through-the-lid implantation. The front plate is usually $5 \mathrm{~mm}$ in diameter and the back plate is $8.5 \mathrm{~mm}$ in diameter. The back plate also has two rows of eight holes, each $1.5 \mathrm{~mm}$ in diameter. The type II Boston KPro is reserved for extreme dry eye conditions and end-stage ocular surface diseases with significant cicatricial conjunctival changes, such as mucous membrane pemphigoid (MMP) and Stevens Johnson syndrome (SJS), in which there is a lack of fornices to support a contact lens as recommended for the type I device. Both types are custom made to have a range of dioptric powers to match the axial length of the patient's eye when aphakic (the natural lens, if present, is removed during surgery and no additional intraocular lens needs to be implanted). 
Other artifical corneas such as AlphaCor, osteo-odonto-keratoprosthesis, and FyodorovZuev keratoprosthesis, work in the same general manner, as substitutes for donor corneas.

\section{Why it is important to do this review}

There has been a renewed interest in artificial corneal implantation following the approval of AlphaCor device in 2002 by US Food and Drug Administration (FDA). Although the Boston type I device was cleared by US FDA in 1992, prior to 2004, fewer than 100 had been implanted, most of which were performed at the Massachusetts Eye and Ear Infirmary (the distributor of the device) (Zerbe 2006). This renewed interest resulted in a wealth of studies evaluating artificial corneal devices. However, due to high complication rates associated with the AlphaCor, the device has been removed from the market, leaving the artificial corneal device arena mostly to the Boston KPro. Once considered a last resort, the Boston KPro is now frequently a viable alternative for eyes with prior failure of traditional donor penetrating keratoplasty. Furthermore, there has been interest in expanding indications for KPro implantation as a primary procedure in patients with limbal stem cell failure from various causes (Michael 2008; Utine 2011). As surgeons and centers have gained more experience with keratoprosthesis, it has become apparent that artificial corneal devices may be an alternative to repeat PK in a broader subset of patients than previously considered. It also has been suggested that artificial corneal transplant surgery is comparable to PK surgery using donor corneas in terms of cost-effectiveness (Ament 2010). The purpose of this review was to compare systematically the clinical performance of artificial corneas with the current standard of care, transplantation with donor corneas, as the use of keratoprostheses is becoming more popular for repeat corneal replacement procedures.

\section{OBJECTIVES}

To assess the effectiveness of artificial versus donor corneas in individuals who have had one or more failed donor corneal transplantations.

\section{METHODS}

\section{Criteria for considering studies for this review}

Types of studies-We planned to include randomized controlled trials (RCTs) and quasirandomized controlled clinical trials (CCTs). We also stated a priori that we would discuss findings from non-RCTs, prospective and retrospective cohort studies, and interventional case series (Akpek 2012). We excluded from our discussion of the latter group of studies those that reported on fewer than 10 individuals/cases. We grouped multiple reports from the same study when authors reported only information on the same group of participants (e.g. results from longer follow-up, data for subsets of participants).

Types of participants-We included studies of participants with corneal opacity who had failed one or more full thickness PKs. We excluded reports of primary keratoprosthesis cases only. We included studies with adults (ages 18 years and older), but did not exclude studies that also included some participants less than 18 years of age. However, we excluded the studies that reported results exclusively or mostly from pediatric patients. There was no restriction regarding whether participants were phakic, aphakic, or pseudophakic. 
Types of interventions-We included artificial corneas (keratoprostheses) of any type and full thickness, and penetrating human donor corneal transplantations. We excluded studies of anterior or posterior (endothelial) lamellar corneal transplantations for previous graft failures. We also reported each type of artificial cornea (e.g. Boston KPro, AlphaCor, osteo-odonto-keratoprosthesis, and Fyodorov-Zuev KPro) individually.

\section{Types of outcome measures}

Primary outcomes: The primary outcome for comparison of corneal transplant procedures was the proportion of participants with best-corrected visual acuity (BCVA) of 20/100 or better, measured as Snellen equivalent, at two years after corneal replacement.

Secondary outcomes: Secondary outcomes for comparison of corneal transplant procedures included:

- the proportion of participants with BCVA of 20/100 or better at one and five years after corneal replacement;

- the proportion of participants with worse vision than pre-operative vision at one, two and five years after corneal replacement;

- the mean change in BCVA at one, two and five years after corneal replacement;

- the proportion of participants with corneal graft failure at one, two and five years after corneal replacement;

- PK group: the proportion of participants with corneal allograft rejection or failure leading to opacity of the graft at one, two and five years after corneal replacement;

- KPro group: the proportion of participants with removal of KPro due to any cause at one, two and five years after corneal replacement;

- the proportion of participants with enucleation of the eyeball due to any cause at one, two and five years after corneal replacement.

When data were available, we reported the proportion of participants with failure who required another corneal surgery with a donor or artificial cornea, and the proportion of participants who had complications requiring other surgery, such as glaucoma and retinal detachment.

We also summarized adverse events reported by the individual studies, including glaucoma, infection, retinal detachment, retroprosthetic membrane formation and further extrusion of the device. We planned to compare quality of life outcomes and economic outcomes; however, these outcomes were not reported by eligible studies.

\section{Search methods for identification of studies}

Electronic searches-We searched CENTRAL (which contains the Cochrane Eyes and Vision Group Trials Register) (2013, Issue 10), Ovid MEDLINE, Ovid MEDLINE InProcess and Other Non-Indexed Citations, Ovid MEDLINE Daily, Ovid OLDMEDLINE (January 1946 to November 2013), EMBASE (January 1980 to November 2013), Latin 
American and Caribbean Health Sciences Literature Database (LILACS) (January 1982 to November 2013), the metaRegister of Controlled Trials ( $m$ RCT) (www.controlledtrials.com), ClinicalTrials.gov (www.clinicaltrials.gov) and the World Health Organization (WHO) International Clinical Trials Registry Platform (ICTRP) (www.who.int/ictrp/search/ en). We did not use any date or language restrictions in the electronic searches for trials. We last searched the electronic databases on 27 November 2013.

See: Appendices for details of search strategies for CENTRAL (Appendix 1), MEDLINE (Appendix 2), EMBASE (Appendix 3), LILACS (Appendix 4), mRCT (Appendix 5), ClinicalTrials.gov (Appendix 6) and the ICTRP (Appendix 7).

Searching other resources-We searched the reference lists of relevant studies for any additional studies not identified by the electronic searches. We planned to use the Science Citation Index to identify potentially relevant studies that cited included studies; however, since no RCTs or CCTs were included, we did not use this database. We contacted experts in the field for information on current, past or unpublished trials, but no RCTs or CCTs were identified (KPro Study Group Bibliography 2013).

\section{Data collection and analysis}

Selection of studies-Two pairs of review authors (SN and FH; KL and MA) assessed the search results independently. Authors classified each record based on title and abstract as: (1) definitely relevant, (2) possibly relevant, or (3) definitely not relevant according to the 'Criteria for considering studies for this review'. No relevant RCTs or CCTs were identified; thus, we classified no records as (1) definitely relevant. We resolved discrepancies by consensus for non-randomized studies assessed as (2) possibly relevant. For consideration of non-randomized studies to include in discussion, we obtained full text copies of reports and two authors working independently assessed each as (a) include for discussion, (b) exclude from discussion, or (c) exclude, not relevant. Studies assessed as (a) include for discussion are listed in the review as Excluded studies. Studies assessed as (b) exclude from discussion or (c) exclude, not relevant are summarized, but not listed individually in the Results section under 'Excluded studies'. We resolved discrepancies by consensus. No study was classified as unclear after review of the full text and there were no study reports in languages that we required to be translated.

Data extraction and management-Two pairs of review authors ( $\mathrm{SN}$ and $\mathrm{FH}$; KL and MA) extracted data independently onto data extraction forms adapted from Cochrane Eyes and Vision Group forms. Study characteristics extracted for each relevant study included methods, participants, interventions, outcomes, and funding sources. One review author entered the data into Review Manager (RevMan 2014) and a second review author verified the data entered. We resolved discrepancies by discussion. We did not contact primary investigators of observational studies to request missing data; instead we used the information as available in the published reports.

Assessment of risk of bias in included studies-Two review authors assessed independently the sources of systematic bias in studies according to the methods described 
in Chapter 8 (Higgins 2011) and Chapter 13 (Reeves 2011) of the Cochrane Handbook for Systematic Reviews of Interventions. We resolved discrepancies through discussion.

We planned to consider the following parameters when assessing risk of bias in RCTs and CCTs: (a) selection bias (random sequence generation, quality of allocation concealment); (b) detection bias (masking of outcome assessors and data analyzers); (c) attrition bias (completeness of follow-up, intention-to-treat (ITT) analysis); (d) reporting bias; and (e) other potential sources of bias (such as funding source). We did not plan to assess masking (blinding) of study participants and personnel (performance bias) due to the differences in interventions and surgical procedures. Each risk of bias parameter was to be assessed as having a "low risk of bias", a "high risk of bias" or an "unclear risk of bias" (insufficient information to permit judgment of low or high risk).

Although a formal assessment of risk of bias was not done for observational studies, we considered the following parameters when discussing cohort studies: (a) selection bias (similarity between groups, reporting of baseline characteristics and potential confounders); (b) performance bias (objective measurements of exposures); (c) detection bias (masking of outcome assessors, objective measurements of outcomes, equal likelihood of detecting outcome for both groups); (d) attrition bias (low attrition rates, similar follow-up between groups); (e) reporting bias; and (f) other potential sources of bias (such as funding source).

Risk of bias considerations for non-comparative studies included: (a) selection bias (consecutive versus preferential selection of participants); (b) performance bias and detection bias (objective measurements of exposures and outcomes); (c) attrition bias (rate of loss to follow-up); (d) reporting bias; and (e) other potential sources of bias (such as funding source).

Measures of treatment effect-The primary outcome for this review was a dichotomous outcome. We planned to report the measure of effect as a risk ratio with $95 \%$ confidence intervals. We planned to report dichotomous secondary outcomes in the same manner.

We planned to report mean changes in BCVA as mean differences between groups with $95 \%$ confidence intervals. We also planned to report continuous data for quality of life outcomes or economic outcomes as mean differences with $95 \%$ confidence intervals.

Unit of analysis issues-The unit of analysis was the eye. For studies in which both eyes of a single participant were included, we reported whether or not adjustments for interperson correlation of outcomes were made.

Dealing with missing data-No RCTs or CCTs were included in the review and we did not contact study authors of non-randomized studies for additional information.

Assessment of heterogeneity-We planned to use the $\mathrm{I}^{2}$ statistic to examine heterogeneity. An $\mathrm{I}^{2}$ value greater than $60 \%$ would have been interpreted as indicating substantial statistical heterogeneity. If substantial statistical heterogeneity was present, we did not plan to conduct meta-analysis and instead would have reported the study results 
independently. We also planned to assess clinical heterogeneity based on the characteristics of participants in the included studies, including type of artificial cornea, lens status (phakic, aphakic, or pseudophakic), age, and underlying co-morbidities (such as retinal detachment, glaucoma, and ocular surface disease).

Assessment of reporting biases-We planned to examine the symmetry of funnel plots to assess reporting biases when more than 10 studies were included in a meta-analysis.

Data synthesis-We did not perform quantitative data synthesis.

Subgroup analysis and investigation of heterogeneity-We did not perform subgroup analysis.

Sensitivity analysis-We did not perform sensitivity analysis.

\section{RESULTS}

\section{Description of studies}

Results of the search-We identified a total of 853 unique records by the electronic searches as of 27 November 2013 (Figure 1). After screening of titles and abstracts, we did not identify any relevant RCTs or CCTs comparing artificial versus donor corneas in people undergoing repeat corneal transplantation. Of the 853 records from the search, we excluded 690 records and reviewed full-text reports for 163 potentially relevant records. From the 163 potentially relevant records, we identified 41 reports of 19 non-randomized studies that evaluated keratoprosthesis in people undergoing repeat corneal transplantation, which we assessed as relevant for discussion.

Non-randomized studies-Of the 19 studies of keratoprosthesis we identified, 14 evaluated the Boston KPro (previously known as the Dohlman-Doane KPro), three AlphaCor (previously known as the Chirila KPro); and one each of osteo-odontokeratoprosthesis (OOKP) and Fyodorov-Zuev keratoprosthesis (see Characteristics of excluded studies).

Boston KPro: The Boston Type 1 KPro Study Group consisted of 19 surgeons at 18 medical centers who volunteered clinical data of Boston keratoprosthesis type 1 surgeries they performed in 300 eyes of 300 patients between January 2003 and July 2008 (Boston Type1 KPro Study). Some of these surgeons also have published separate reports of outcomes from their own patients, which made it difficult to assess study cohorts independently. In addition to the Boston Type 1 KPro Study, we identified case series reports from 12 individual institutions (combined total of 638 patients; 687 eyes) and one international case series (100 patients; 107 eyes) (Table 1).

Visual acuity and graft failure outcomes were reported in many of the Boston KPro studies. However, few studies reported outcomes at specified follow-up times (e.g. one year, two years, five years) (Table 2). These studies also had large amounts of missing data, particularly beyond one year of follow-up. 
AlphaCor: Similar to the Boston Type 1 KPro Study Group, Hicks and colleagues compiled clinical data of AlphaCor surgeries between 1998 to 2006 from surgeons who voluntarily contributed their patient data (Hicks 2006). This case series included data reported by 84 surgeons from 11 countries who implanted 322 AlphaCor devices in 302 patients. We identified two additional case series of Alpha-Cor (Table 3).

Visual acuity data were reported in all of the three AlphaCor studies; however only one study reported outcomes at specified follow-up times (Table 3). Two studies reported graft failure or retention outcomes.

Other types of artificial corneas: We identified case series reports of two other types of artificial corneal devices, one each of Fyodorov-Zuev keratoprosthesis (10 patients) and osteo-odonto-keratoprosthesis (OOKP) (25 patients). Both studies reported visual acuity outcomes and number of device extrusions (Table 4).

Excluded studies-We further excluded 122 reports after review of the full-text for the following reasons: 47 reports were of primary KPro procedures or did not specify how many patients had previous PK procedures; 31 reports included fewer than 10 patients undergoing repeat corneal transplantation; 34 reports did not include original data (e.g. editorials, descriptions of surgical procedures, reviews); and 10 reports dealt with postoperative management of corneal transplantation (e.g. use of soft contact lenses, how to image eyes with implanted KPros, treatment of endophthalmitis).

\section{Risk of bias in included studies}

We could not assess risk of bias since no RCTs were included in the review.

\section{Effects of interventions}

We did not identify any evidence comparing the effectiveness of artificial versus donor corneas for repeat corneal transplantation.

\section{DISCUSSION}

\section{Summary of main results}

No relevant randomized or quasi-randomized controlled trials were identified for inclusion in this review. We identified 19 studies of KPro surgery in patients undergoing repeat corneal transplantation, none of which compared implantation of KPro devices with human donor corneal transplantation.

The majority of non-randomized studies we identified were reports of case series of KPro implantations from medical centers in the USA. One study compared results of Boston KPros implanted at a medical center in the USA with a case series of Boston KPros implanted internationally (Aldave 2012a; Aldave 2012b). The authors of the report concluded that visual acuity outcomes, retention rates, and adverse events were comparable between the two groups of patients. It is important to note, however, that the average followup times differed in the two cohorts, with the US-based cohort having a mean follow-up of 
24 months (up to 84 months) and the non-US-based group having a mean follow-up of 14 months (up to 48 months).

When data could be abstracted from the non-randomized studies, the failure rates at one year after implantation ranged from $0 \%$ to $30 \%$. However, these rates are very uncertain due to the high amount of loss to follow-up in the studies and the variability in patient risk factors (e.g. indication for surgery, number of previous graft failures).

\section{Overall completeness and applicability of evidence}

This review aimed to gain information regarding the visual outcomes and complication rates of artificial corneal transplantation surgeries in comparison with repeat donor corneal transplantation in individuals with prior failed full thickness penetrating keratoplasty. No such studies directly comparing the results were noted from extensive literature searches. Given the increasing popularity of Boston type I KPro and a trend to expanding the indications even to patients who have not yet received donor transplantations, a head-tohead comparison is needed. The inclusion criteria to enroll participants in such a study should be carefully considered. For instance, a non-vascularized cornea in a phakic eye after a single episode of rejection may do very well with repeat donor grafting and probably would not be considered for KPro surgery. The situation is entirely different when there are additional risk factors for failure such as neovascularization, ocular surface diseases, or glaucoma. Future studies comparing these methods should take into consideration the cause of prior graft failure, preoperative diagnosis, and presence of high risk characteristics for rejection when enrolling participants.

In addition, the currently available studies reporting outcomes of both repeat donor PK as well as KPro surgeries are limited in regards to the length of follow-up. It is conceivable that the lifetime risks of complications and the possibility of permanent vision loss after either procedure may differ significantly. This fact could not be addressed in this review due to the very limited follow-up of the available reports. A careful review of literature of repeat PK outcomes might perhaps help determine the best outcomes to assess in a head-to-head comparison. For example, incidence of endophthalmitis and vision loss due to glaucoma, which have been reported to be higher after implantation of Boston type 1 KPro compared with PK, would be important outcomes to study.

\section{Quality of the evidence}

The literature we found on this topic consisted primarily of clinical studies evaluating only one type of procedure (KPro or PK), i.e. non-comparative case series and case reports. We also found that few of these studies reported visual acuity or graft survival outcomes up to one year of follow-up for all study participants. The most common method of reporting outcomes was to combine participant data at 'final follow-up', rather than at specific followup time points. Since graft survival is associated with the duration of the implantation (i.e. the longer a person has the device), it is inappropriate to combine findings from different follow-up times. When outcomes were reported at specific time points (e.g. six months, one year, two years), data often were not reported for all study participants and reported only for those with available data. 


\section{Potential biases in the review process}

We conducted a highly sensitive search to identify trials comparing artificial corneal implants with human donor corneas for people needing repeat corneal transplantation. As we anticipated finding few or no randomized controlled trials on this topic, we also searched for any observational or clinical study evaluating KPro for repeat corneal transplantation. Clinicians and methodologists on the review team were paired to duplicate tasks in order to minimize selection bias and errors during the study selection and data abstraction processes.

Not all of the studies included only eyes undergoing repeat corneal surgery. Many studies also included keratoprosthesis as a primary procedure in eyes at high risk of failure with donor corneal transplantation (e.g. people with chemical injury, SJS, or MMP). We limited our review of non-randomized studies to those in which more than $60 \%$ of participants had at least one failed PK. We allowed a mix of cases based on clinical experience, knowing these cases were likely to be grouped together in study cohorts.

\section{Agreements and disagreements with other studies or reviews}

We are unaware of any other systematic review comparing artificial versus donor corneas in people needing repeat corneal transplantation following a failed full thickness PK. Based on non-randomized studies identified from our searches, different types of KPros are being used for individuals undergoing repeat corneal transplantation procedures. Historically, artificial corneas have been considered for 'salvage' procedures when no alternative exists. Therefore, the majority of the patients in the published reports had been deemed "ineligible" to receive another donor corneal transplantation and hence were likely to have worse vision prior to the surgery as well as worse co-morbidity than the patients who were considered for repeat corneal transplantation with donor corneas. Despite this trend, the high retention rates reported with Boston type 1 KPro have made this device an attractive alternative to repeat donor corneal transplantations (AAO PPP 2013; Boston Type 1 KPro Study). As surgeons and centers have gained more experience with KPro, particularly following advances in design and materials, the utilization of KPros has shown a substantial increase in the most recent few years including in a wider subset of patients than previously considered (MEEI 2013).

Importantly, the number of repeat corneal transplantations in the US has been steadily increasing. According to the Eye Bank Association of America, repeat corneal transplantation for a previously failed graft is the second most common indication for full thickness donor PK in the US, after keratoconus, comprising about $17 \%$ of all those undergoing a full thickness donor PK during the last decade (EBAA 2013). This percentage is in line with rates from other countries as well (Bersudsky 2001; Wagoner 2009; Williams 1995). Of all transplant procedures, corneal transplantation has a good success rate: however, the risk of graft failure increases with the number of repeat procedures and time. The graft survival rate at one year was reported to be $80.5 \%$ (95\% CI $75.4 \%$ to $85.0 \%$ ) for the first repeat PK, $76.9 \%$ (95\% CI 64.9\% to 85.6\%) for the second repeat PK, and 51.9\% (95\% CI 33.4\% to $73.4 \%$ ) for the third or more repeat PKs based on national data from Australia (ACGR 1993). A more recent study of repeat PK in the USA showed better overall survival rates for the second or more repeat PKs than the Austrailian study, but also 
supported findings that graft survival at one year was higher for eyes having their second repeat PK $(89 \%, 95 \%$ CI $76 \%$ to $96 \%)$ versus their third repeat PK $(73 \%, 95 \%$ CI $39 \%$ to 94\%) (Yildiz 2010).

\section{AUTHORS' CONCLUSIONS}

\section{Implications for practice}

The optimal management for patients who have failed conventional corneal transplantation is unknown. Our review of the evidence did not discover any comparative clinical studies evaluating artificial corneal devices versus donor corneas in patients who had failed one or more full thickness human corneal transplantations. Currently, there are no guidelines regarding the limit to the number of times donor corneal transplantation should be repeated. The corneal surgeons managing these patients are left to make recommendations based only on patient values, personal clinical experience, local culture, and non-randomized studies.

\section{Implications for research}

Currently, in some centers, KPros routinely are recommended after a single graft failure; in others, not until after multiple graft failures or not at all. To date, there have been no controlled trials comparing the visual outcomes and complications of artificial corneal devices with repeat donor corneal transplantation to guide surgeons and their patients despite wide use of the Boston type 1 KPro and other artificial corneal devices. It is apparent that such a trial is needed and should be conducted before an approach is adopted without evidence of comparative effectiveness. The findings from a randomized controlled trial would offer significant benefit to an ever-increasing pool of people with visual disability due to corneal opacification, most of whom are still in productive stages of their lives. It is important that future studies measure clinical and patient-important outcomes at specified follow-up time points and account for all study participants in the analyses. Of particular interest would be long-term visual acuity outcomes and complications (e.g., two and five years post-transplantation) to help inform the expected benefits and risks associated with each procedure over time. Cost comparison outcomes also should be considered to evaluate the applicability of these procedures, particularly in regions with few eye banking resources.

\section{Acknowledgments}

We acknowledge Iris Gordon, Trials Search Co-ordinator for the Cochrane Eyes and Vision Group (CEVG), for devising and running electronic search strategies. We acknowledge Anthony Aldave, Roy Chuck, and Anupa Shah (Managing Editor for CEVG) for commenting on previous versions of this review. We thank Irmgard Behlau, Maria Cortina, Francis Price, Marie-Claude Robert and Barbara Hawkins for reviewing and providing feedback for this review.

\section{SOURCES OF SUPPORT}

Internal sources

- No sources of support supplied

External sources

- Grant 1 U01 EY020522, National Eye Institute, National Institutes of Health, USA.

- National Institute for Health Research (NIHR), UK. 
- Richard Wormald, Co-ordinating Editor for the Cochrane Eyes and Vision Group (CEVG) acknowledges financial support for his CEVG research sessions from the Department of Health through the award made by the National Institute for Health Research to Moorfields Eye Hospital NHS Foundation Trust and UCL Institute of Ophthalmology for a Specialist Biomedical Research Centre for Ophthalmology.

- The NIHR also funds the CEVG Editorial Base in London.

The views expressed in this publication are those of the authors and not necessarily those of the NIHR, NHS, or the Department of Health.

\author{
Appendix 1. CENTRAL search strategy \\ \#1 keratoprostheses or keratoprosthesis \\ \#2 Kpro \\ \#3 AlphaCor \\ \#4 (\#1 OR \#2 OR \#3)
}

\title{
Appendix 2. MEDLINE (OVID) search strategy
}
1. exp animals/
2. exp humans/
3. 1 not (1 and 2)
4. (keratoprostheses or keratoprosthesis).tw.
5. Kpro.tw.
6. AlphaCor.tw.
7. or/4-6
8. 7 not 3

\section{Appendix 3. EMBASE (OVID) search strategy}

1. exp animals/

2. exp humans/

3. 1 not (1 and 2$)$

4. keratoprosthesis/

5. (keratoprostheses or keratoprosthesis).tw

6. Kpro.tw.

7. AlphaCor.tw.

8. or $/ 4-7$

9. 8 not 3 


\section{Appendix 4. LILACS search strategy}

keratoprostheses or keratoprosthesis or Kpro or AlphaCor

\section{Appendix 5. metaRegister of Controlled Trials search strategy}

keratoprostheses or keratoprosthesis or Kpro or AlphaCor

\section{Appendix 6. ClinicalTrials.gov search strategy}

Keratoprostheses OR Keratoprosthesis OR Kpro OR AlphaCor

\section{Appendix 7. ICTRP search strategy}

Keratoprostheses OR Keratoprosthesis OR Kpro

\section{DATA AND ANALYSES}

This review has no analyses.

\section{DIFFERENCES BETWEEN PROTOCOL AND REVIEW}

Because no eligible trials were identified after screening titles and abstracts, we revised the classification of studies for the full-text screening stage. Rather than classifications of (a) include, (b) unclear, or (c) exclude, we assessed study reports as (a) include for discussion, (b) exclude from discussion, or (c) exclude, not relevant. Studies assessed as (a) include for discussion are listed in the review as Excluded studies.

\section{References to studies excluded from this review}

Aldave 2012a \{published data only\}. Aldave AJ, Kamal KM, Vo RC, Yu F. The Boston type I keratoprosthesis: improving outcomes and expanding indications. Ophthalmology. 2009; 116(4): 640-51. [PubMed: 19243830]

Aldave AJ, Sangwan VS, Basu S, Basak SK, Hovakimyan A, Gevorgyan O, et al. International results with the Boston type I keratoprosthesis. Ophthalmology. 2012; 119(8):1530-8. [PubMed: 22512986]

Goldman DR, Hubschman JP, Aldave AJ, Chiang A, Huang JS, Bourges JL, et al. Postoperative posterior segment complications in eyes treated with the Boston type I keratoprosthesis. Retina. 2013; 33(3):532-41. [PubMed: 23073339]

Kim MJ, Yu F, Aldave AJ. Microbial keratitis after Boston type I keratoprosthesis implantation: incidence, organisms, risk factors, and outcomes. Ophthalmology. 2013; 120(11):2209-16. [PubMed: 23747162]

Sejpal K, Yu F, Aldave AJ. The Boston keratoprosthesis in the management of corneal limbal stem cell deficiency. Cornea. 2011; 30(11):1187-94. [PubMed: 21885964]

Aldave 2012b \{published data only\}. Aldave AJ, Sangwan VS, Basu S, Basak SK, Hovakimyan A, Gevorgyan O, et al. International results with the Boston type I keratoprosthesis. Ophthalmology. 2012; 119(8):1530-8. [PubMed: 22512986]

Aquavella 2005 \{published data only\}. Aquavella JV, Qian Y, McCormick GJ, Palakuru JR. Keratoprosthesis: current techniques. Cornea. 2006; 25(6):656-62. [PubMed: 17077656]

Aquavella JV, Qian Y, McCormick GJ, Palakuru JR. Keratoprosthesis: the Dohlman-Doane device. American Journal of Ophthalmology. 2005; 140(6):1032-8. [PubMed: 16376647] 
Boston Type 1 KPro Study \{published data only . Ament JD, Stryjewski TP, Ciolino JB, Todani A, Chodosh J, Dohlman CH. Cost-effectiveness of the Boston keratoprosthesis. American Journal of Ophthalmology. 2010; 149(2):221-8. [PubMed: 19939347]

Ciolino JB, Belin MW, Todani A, Al-Arfaj K, Rudnisky CJ. Boston Type 1 Keratoprosthesis Study Group. Retention of the Boston keratoprosthesis type 1: multicenter study results. Ophthalmology. 2013; 120(6):1195-200. [PubMed: 23499061]

Rudnisky CJ, Belin MW, Todani A, Al-Arfaj K, Ament JD, Zerbe BJ, et al. Risk factors for the development of retroprosthetic membranes with Boston keratoprosthesis type 1: multicenter study results. Ophthalmology. 2012; 119(5):951-5. [PubMed: 22361316]

Zerbe BL, Belin MW, Ciolino JB. Boston Type 1 Keratoprosthesis Study Group. Results from the multicenter Boston Type 1 Keratoprosthesis Study. Ophthalmology. 2006; 113(10):1779.e1-7. [PubMed: 16872678]

Chew 2009 \{published data only\}. Chew HF, Ayres BD, Hammersmith KM, Rapuano CJ, Laibson PR, Myers JS, et al. Boston keratoprosthesis outcomes and complications. Cornea. 2009; 28(9): 989-96. [PubMed: 19724214]

Dunlap 2010 \{published data only\}. Akpek EK, Harissi-Dagher M, Petrarca R, Butrus SI, Pineda R 2nd, Aquavella JV, et al. Outcomes of Boston keratoprosthesis in aniridia: a retrospective multicenter study. American Journal of Ophthalmology. 2007; 144(2):227-31. [PubMed: 17543875]

Dunlap K, Chak G, Aquavella JV, Myrowitz E, Utine CA, Akpek E. Short-term visual outcomes of Boston type 1 keratoprosthesis implantation. Ophthalmology. 2010; 117(4):687-92. [PubMed: 20096462]

Khan BF, Harissi-Dagher M, Pavan-Langston D, Aquavella JV, Dohlman CH. The Boston keratoprosthesis in herpetic keratitis. Archives of Ophthalmology. 2007; 125(6):745-9. [PubMed: 17562983]

Ghaffariyeh 2011 \{published data only\}. Ghaffariyeh A, Honarpisheh N, Karkhaneh A, Abudi R, Moroz ZI, Peyman A, et al. Fyodorov-Zuev keratoprosthesis implantation: long-term results in patients with multiple failed corneal grafts. Graefes Archive for Clinical and Experimental Ophthalmology. 2011; 249(1):93-101.

Greiner 2011 \{published data only\}. Bradley JC, Hernandez EG, Schwab IR, Mannis MJ. Boston type 1 keratoprosthesis: the University of California Davis experience. Cornea. 2009; 28(3):321-7. [PubMed: 19387235]

Greiner MA, Li JY, Mannis MJ. Longer-term vision outcomes and complications with the Boston type 1 keratoprosthesis at the University of California, Davis. Ophthalmology. 2011; 118(8):1543-50. [PubMed: 21397948]

Shapiro BL, Cortes DE, Chin EK, Li JY, Werner JS, Redenbo E, et al. High-resolution spectral domain anterior segment optical coherence tomography in type 1 Boston keratoprosthesis. Cornea. 2013; 32(7):951-5. [PubMed: 23591146]

Guell JL, Arcos E, Gris O, Aristizabal D, Pacheco M, Sanchez CL, et al. Outcomes with the Boston Type 1 Keratoprosthesis at Instituto de Microcirugia Ocular IMO. Saudi Journal of Ophthalmology. 2011; 25(3):281-4. [PubMed: 23960937]

Guell 2011 \{published data only\}. Verdejo-Gomez L, Pelaez N, Gris O, Guell JL. The Boston Type I keratoprosthesis: an assessment of its efficacy and safety. Ophthalmic Surgery, Lasers and Imaging. 2011; 42(6):446-52.

Harissi-Dagher 2007 \{published data only\}. Harissi-Dagher M, Dohlman CH. The Boston keratoprosthesis in severe ocular trauma. Canadian Journal of Ophthalmology. 2008; 43(2):1659. [PubMed: 18347618]

Harissi-Dagher M, Khan BF, Schaumberg DA, Dohlman CH. Importance of nutrition to corneal grafts when used as a carrier of the Boston keratoprosthesis. Cornea. 2007; 26(5):564-8. [PubMed: 17525653]

Nouri M, Terada H, Alfonso EC, Foster CS, Durand ML, Dohlman CH. Endophthalmitis after keratoprosthesis: incidence, bacterial causes, and risk factors. Archives of Ophthalmology. 2001; 119(4):484-9. [PubMed: 11296013] 
Sayegh RR, Ang LP, Foster CS, Dohlman CH. The Boston keratoprosthesis in Stevens-Johnson syndrome. American Journal of Ophthalmology. 2008; 145(3):438-44. [PubMed: 18207122]

Yaghouti F, Nouri M, Abad JC, Power WJ, Doane MG, Dohlman CH. Keratoprosthesis: preoperative prognostic categories. Cornea. 2001; 20(1):19-23. [PubMed: 11188997]

Hicks 2006 \{published data only\}. Crawford GJ, Hicks CR, Lou X, Vijayasekaran S, Tan D, Mulholland B, et al. The Chirila keratoprosthesis: phase I human clinical trial. Ophthalmology. 2002; 109(5):883-9. [PubMed: 11986092]

Hicks CR, Crawford GJ, Dart JK, Grabner G, Holland EJ, Stulting RD, et al. AlphaCor: Clinical outcomes. Cornea. 2006; 25(9):1034-42. [PubMed: 17133049]

Hicks CR, Crawford GJ, Lou X, Tan DT, Snibson GR, Sutton G, et al. Corneal replacement using a synthetic hydrogel cornea, AlphaCor: device, preliminary outcomes and complications. Eye. 2003; 17(3):385-92. [PubMed: 12724702]

Hicks CR, Crawford GJ, Tan DT, Snibson GR, Sutton GL, Gondhowiardjo TD, et al. Outcomes of implantation of an artificial cornea, AlphaCor: effects of prior ocular herpes simplex infection. Cornea. 2002; 21(7):685-90. [PubMed: 12352086]

Hicks CR, Macvie O, Crawford GJ, Constable IJ. A risk score as part of an evidence-based approach to the selection of corneal replacement surgery. Cornea. 2005; 24(5):523-30. [PubMed: 15968155]

Hille 2006 \{published data only\}. Hille K, Hille A, Ruprecht KW. Medium term results in keratoprostheses with biocompatible and biological haptic. Graefes Archive for Clinical and Experimental Ophthalmology. 2006; 244(6):696-704.

Jiraskova 2011 \{published data only\}. Jiraskova N, Rozsival P, Burova M, Kalfertova M. AlphaCor artificial cornea: clinical outcome. Eye. 2011; 25(9):1138-46. [PubMed: 21681219]

Kamyar 2012 \{published data only\}. Kamyar R, Weizer JS, de Paula FH, Stein JD, Moroi SE, John D, et al. Glaucoma associated with Boston type I keratoprosthesis. Cornea. 2012; 31(2):134-9. [PubMed: 22134402]

Koller 2012 \{published data only\}. Koller B, Neuhann T, Neuhann I. Results with the Boston keratoprosthesis. Ophthalmologe. 2012; 109(5):454-61. [PubMed: 22581046]

Patel 2012 \{published data only\}. Patel AP, Wu EI, Ritterband DC, Seedor JA. Boston type 1 keratoprosthesis: the New York Eye and Ear experience. Eye. 2012; 26(3):418-25. [PubMed: 22173079]

Shihadeh 2012 \{published data only\}. Shihadeh WA, Mohidat HM. Outcomes of the Boston keratoprosthesis in Jordan. Middle East African Journal of Ophthalmology. 2012; 19(1):97-100. [PubMed: 22346122]

Talajic 2012 \{published data only\}. Robert MC, Biernacki K, Harissi-Dagher M. Boston keratoprosthesis type 1 surgery: use of frozen versus fresh corneal donor carriers. Cornea. 2012; 31(4):339-45. [PubMed: 22269678]

Robert MC, Harissi-Dagher M. Boston type 1 keratoprosthesis: the CHUM experience. Canadian Journal of Ophthalmology. 2011; 46(2):164-8. [PubMed: 21708085]

Talajic JC, Agoumi Y, Gagne S, Moussally K, Harissi-Dagher M. Prevalence, progression, and impact of glaucoma on vision after Boston type 1 keratoprosthesis surgery. American Journal of Ophthalmology. 2012; 153(2):267-74. [PubMed: 21982110]

Trichet 2013 \{published data only\}. Trichet E, Carles G, Matonti F, Proust H, Ridings B, Conrath J, et al. Alphacor keratoprosthesis: device, surgical technique and clinical outcomes. Journal Francais d'Opthalmologie. 2013; 36(5):393-401.

\section{Additional references}

AAO PPP 2013. American Academy of Ophthalmology Cornea/External Disease Panel. [6 February 2014] Preferred Practice Pattern ${ }^{\circledR}$ Guidelines. Corneal edema and opacification. www.aao.org/ppp

ACGR 1993. The Australian Corneal Graft Registry. 1990 to 1992 report. Australian and New Zealand Journal of Ophthalmology. 1993; 21(2 Suppl):1-48. [PubMed: 8333942] 
Ament 2010. Ament JD, Stryjewski TP, Ciolino JB, Todani A, Chodosh J, Dohlman CH. Costeffectiveness of the Boston keratoprosthesis. American Journal of Ophthalmology. 2010; 149(2): 221-8. [PubMed: 19939347]

Bersudsky 2001. Bersudsky V, Blum-Hareuveni T, Rehany U, Rumelt S. The profile of repeated corneal transplantation. Ophthalmology. 2001; 108(3):461-9. [PubMed: 11237899]

EBAA 2013. Eye Bank Association of America. [3 February 2014] 2013 Eye banking statistical report. www.restoresight.org/wp-content/uploads/2014/04/2013Statistical.Report-FINAL.pdf

Higgins 2011. Higgins, JPT.; Altman, DG.; Sterne, JAC., editors. Chapter 8: Assessing risk of bias in included studies. Higgins, JPT.; Green, S., editors. Cochrane Handbook for Systematic Reviews of Interventions Version 5.1.0. The Cochrane Collaboration; Available from www.cochranehandbook.org [March 2011]

Ilhan-Sarac 2005. Ilhan-Sarac O, Akpek EK. Current concepts and techniques in keratoprosthesis. Current Opinion in Ophthalmology. 2005; 16(4):246-50. [PubMed: 16000898]

KPro Study Group Bibliography 2013. KPro Study Group. [28 August 2013] Bibliography of keratoprosthesis and artificial cornea and biomaterials therefor 1789 to 2013. www.kpro.org

Ma 2005. Ma JJ, Graney JM, Dohlman CH. Repeat penetrating keratoplasty versus the Boston keratoprosthesis in graft failure. International Ophthalmology Clinics. 2005; 45(4):49-59. [PubMed: 16199966]

MEEI 2013. [23 November 2013] Massachusetts Eye, Ear Infirmary. Boston KPro news. Newsletter IX. 2013. www.masseyeandear.org/gedownload!/Kpro-News-Vol9-Aug-2013.pdf? item.id=74892044\&version.id=74892045

Michael 2008. Michael R, Charoenrook V, de la Paz MF, Hitzl W, Temprano J, Barraquer RI. Longterm functional and anatomical results of osteo- and osteoodonto-keratoprosthesis. Graefes Archive for Clinical and Experimental Ophthalmology. 2008; 246(8):1133-7.

Murthy 2012. Murthy, GVS.; Johnson, GJ. Chapter 1: prevalence, incidence and distribution of visual impairment. In: Johnson, GJ.; Minassian, DC.; Weale, RA.; West, SK., editors. The Epidemiology of Eye Disease. 3. Imperial College Press; 2012.

Reeves 2011. Reeves, BC.; Deeks, JJ.; Higgins, JPT.; Wells, GA. Chapter 13: Including nonrandomized studies. In: Higgins, JPT.; Green, S., editors. Cochrane Handbook for Systematic Reviews of Interventions Version 5.1.0. The Cochrane Collaboration, 2011; updated March 2011 Available from www.cochrane-handbook.org

RevMan 2014. The Nordic Cochrane Centre, The Cochrane Collaboration. Review Manager (RevMan) 5.3. Copenhagen: The Nordic Cochrane Centre, The Cochrane Collaboration; 2014.

Siganos 2010. Siganos CS, Tsiklis NS, Miltsakakis DG, Georgiadis NS, Georgiadou IN, Kymionis GD, et al. Changing indications for penetrating keratoplasty in Greece, 1982-2006: a multicenter study. Cornea. 2010; 29(4):372-4. [PubMed: 20164748]

Thompson 2003. Thompson RW Jr, Price MO, Bowers PJ, Price FW Jr. Long-term graft survival after penetrating keratoplasty. Ophthalmology. 2003; 110(7):1396-402. [PubMed: 12867398]

Todani 2011. Todani A, Ciolino JB, Ament JD, Colby KA, Pineda R, Belin MW, et al. Titanium back plate for a PMMA keratoprosthesis: clinical outcomes. Graefes Archive for Clinical and Experimental Ophthalmology. 2011; 249(10):1515-8.

Utine 2011. Utine CA, Tzu J, Dunlap K, Akpek EK. Visual and clinical outcomes of explantation versus preservation of the intraocular lens during keratoprosthesis implantation. Journal of Cataract and Refractive Surgery. 2011; 37(9):1615-22. [PubMed: 21855761]

Wagoner 2009. Wagoner MD, Gonnah el-S, Al-Towerki AE. King Khaled Eye Specialist Hospital Cornea Transplant Study Group. Outcome of primary adult penetrating keratoplasty in a Saudi Arabian population. Cornea. 2009; 28(8):882-90. [PubMed: 19654527]

Whitcher 2001. Whitcher JP, Srinivasan M, Upadhyay MP. Corneal blindness: a global perspective. Bulletin of the World Health Organization. 2001; 79(3):214-21. [PubMed: 11285665]

Williams 1995. Williams KA, Muehlberg SM, Lewis RF, Coster DJ. How successful is corneal transplantation? A report from the Australian Corneal Graft Register. Eye. 1995; 9(Pt 2):219-27. [PubMed: 7556721] 
Yildiz 2010. Yildiz EH, Hoskins E, Fram N, Rapuano CJ, Hammersmith KM, Laibson PR, et al. Third or greater penetrating keratoplasties: indications, survival, and visual outcomes. Cornea. 2010; 29(3):254-9. [PubMed: 20118784]

Zerbe 2006. Zerbe BL, Belin MW, Ciolino JB. Boston Type 1 Keratoprosthesis Study Group. Results from the multicenter Boston Type 1 Keratoprosthesis Study. Ophthalmology. 2006; 113(10): 1779. [PubMed: 16872678]

\section{References to other published versions of this review}

Akpek $2012 *$. Akpek EK, Alkharashi M, Lindsley K. Artificial corneas versus donor corneas for repeat corneal transplants. Cochrane Database of Systematic Reviews. 2012; (1) Indicates the major publication for the study. 10.1002/14651858.CD009561 


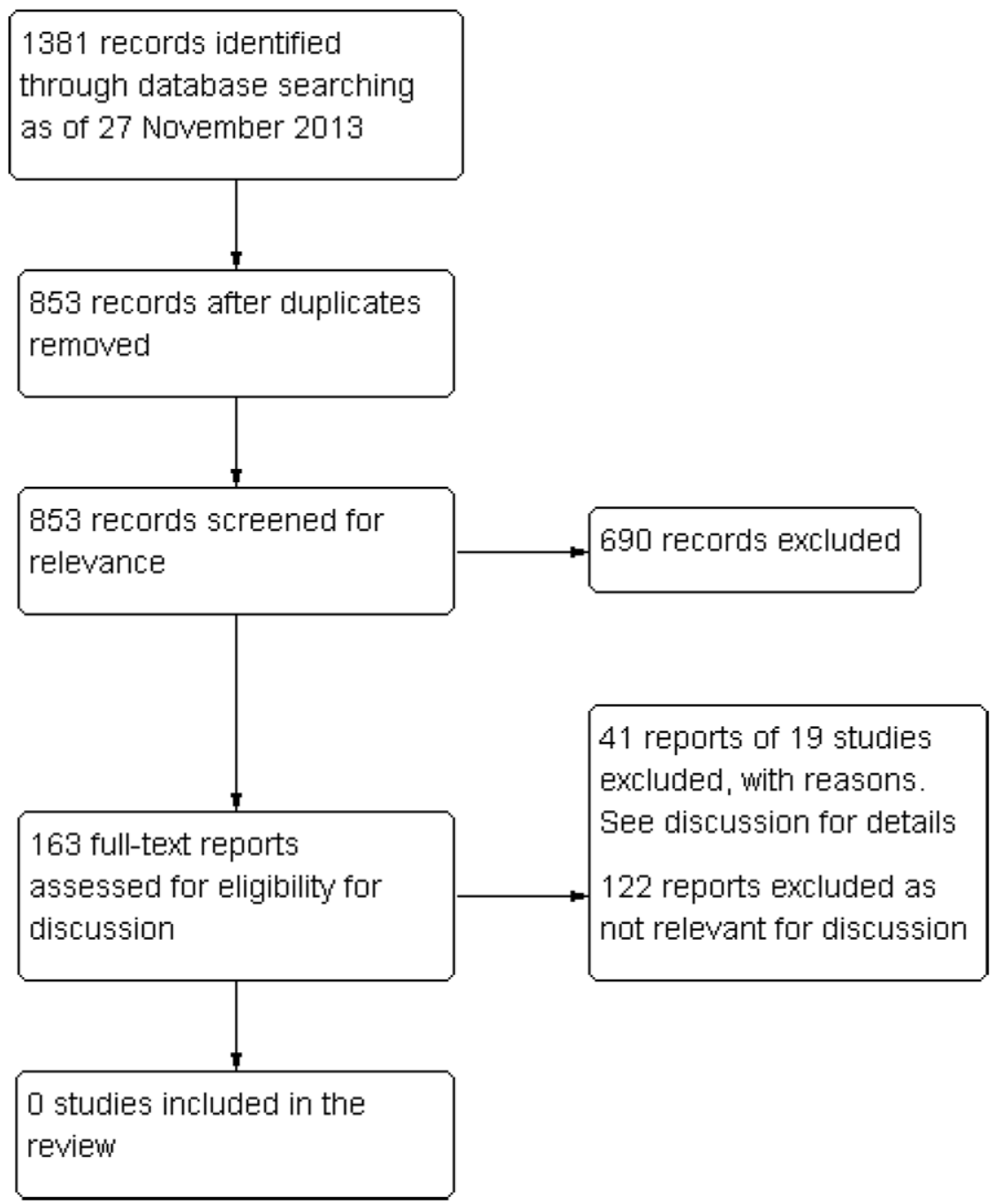

Figure 1. Results for searching for studies for inclusion in the review 


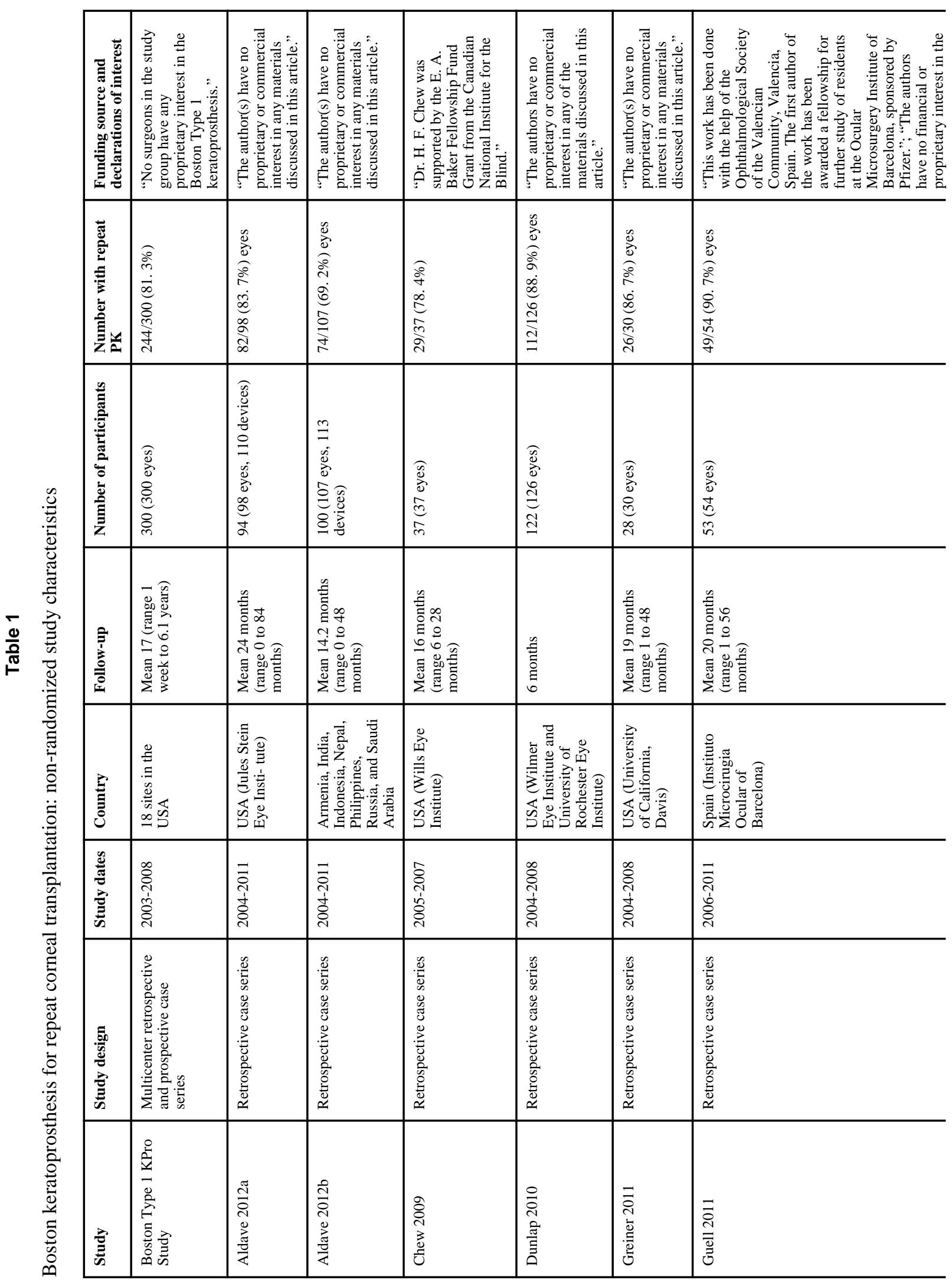




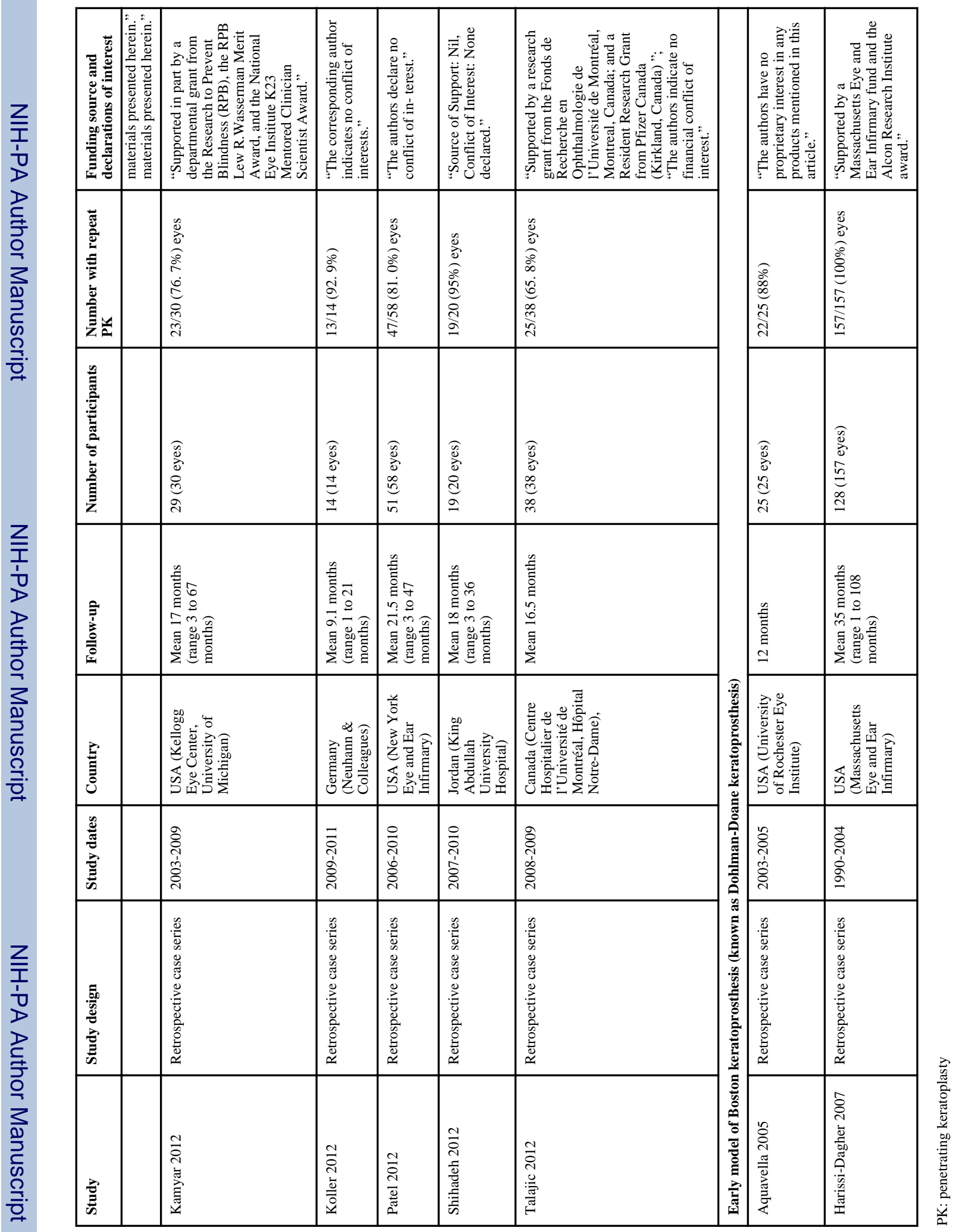




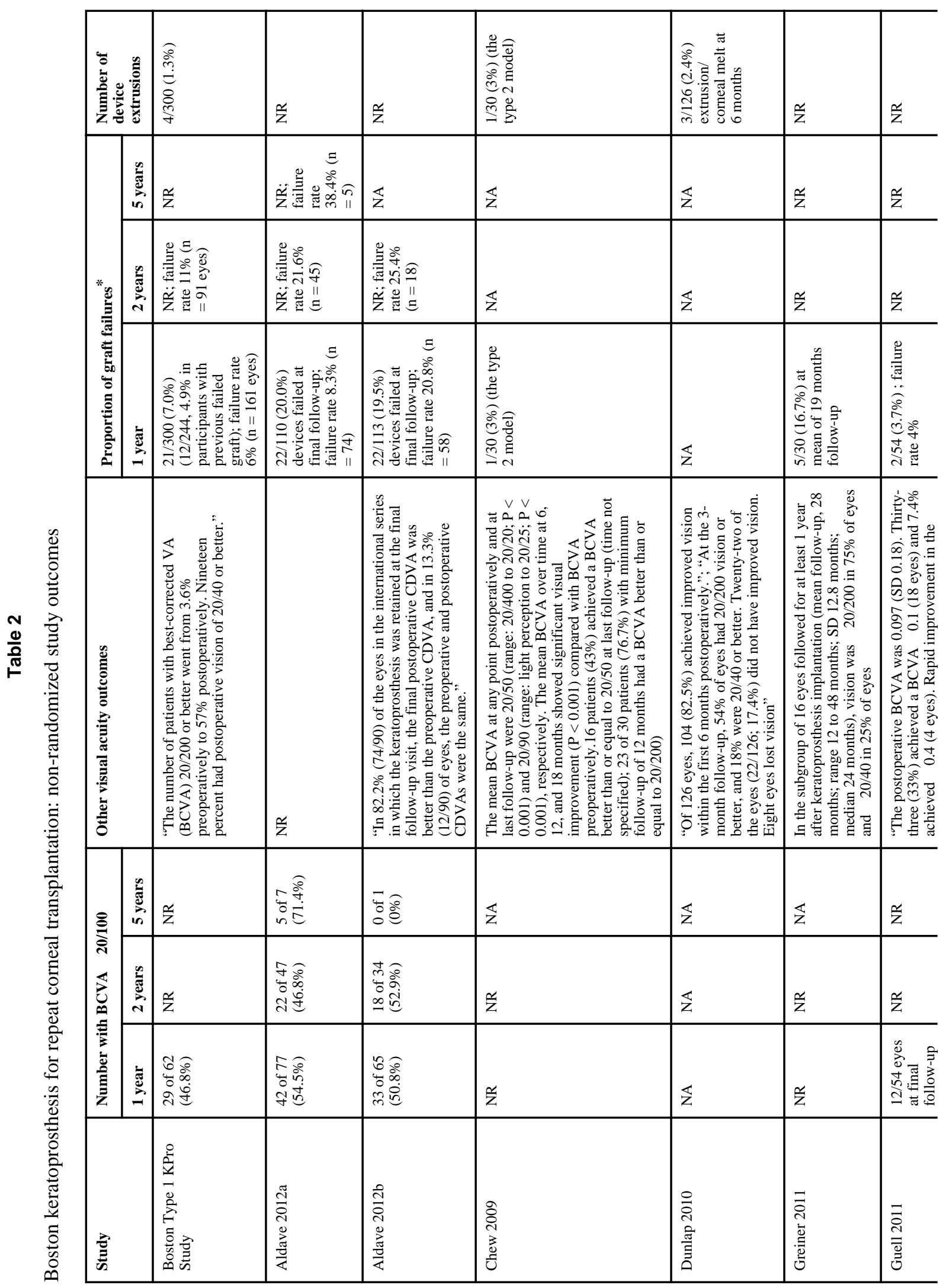




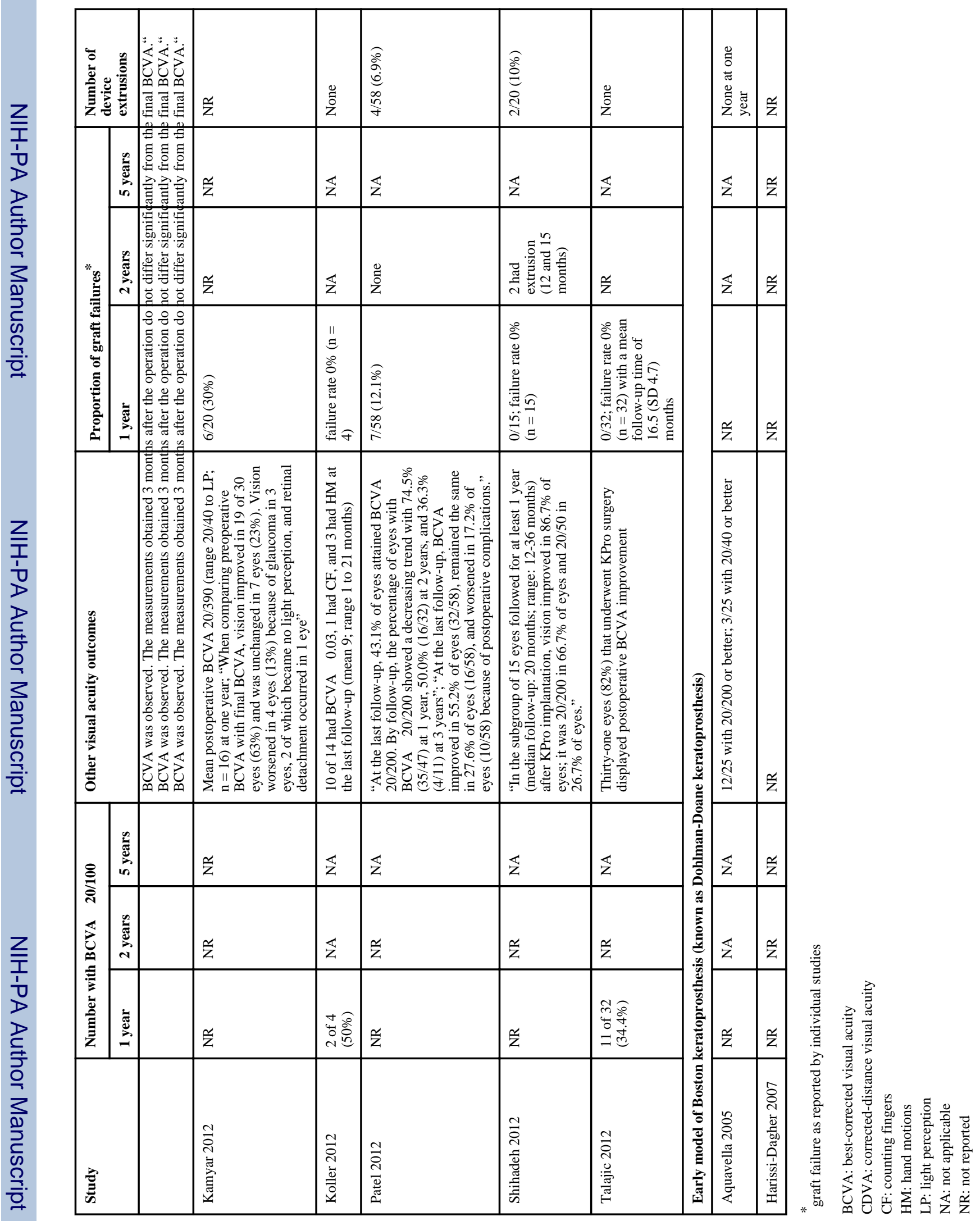



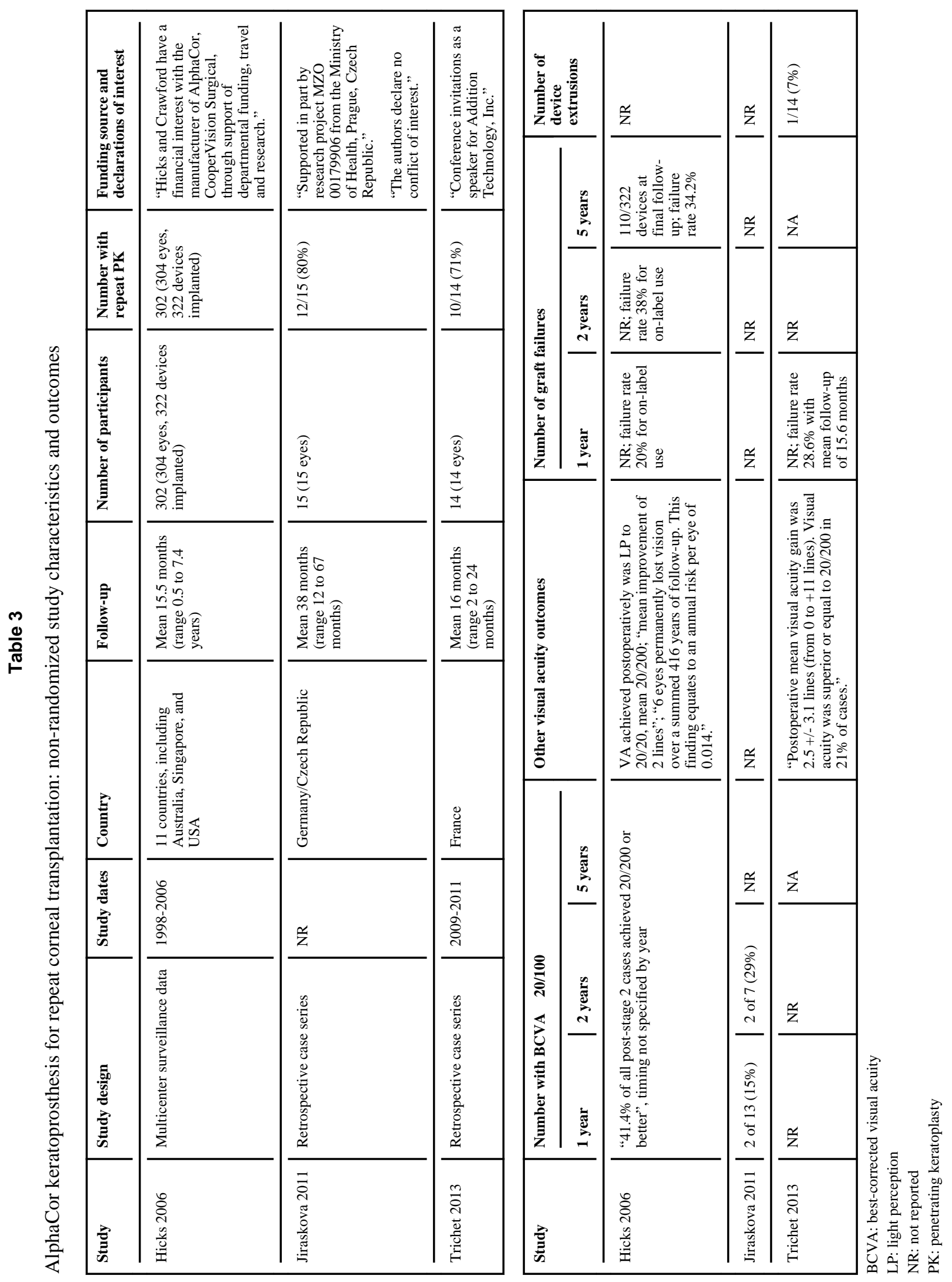

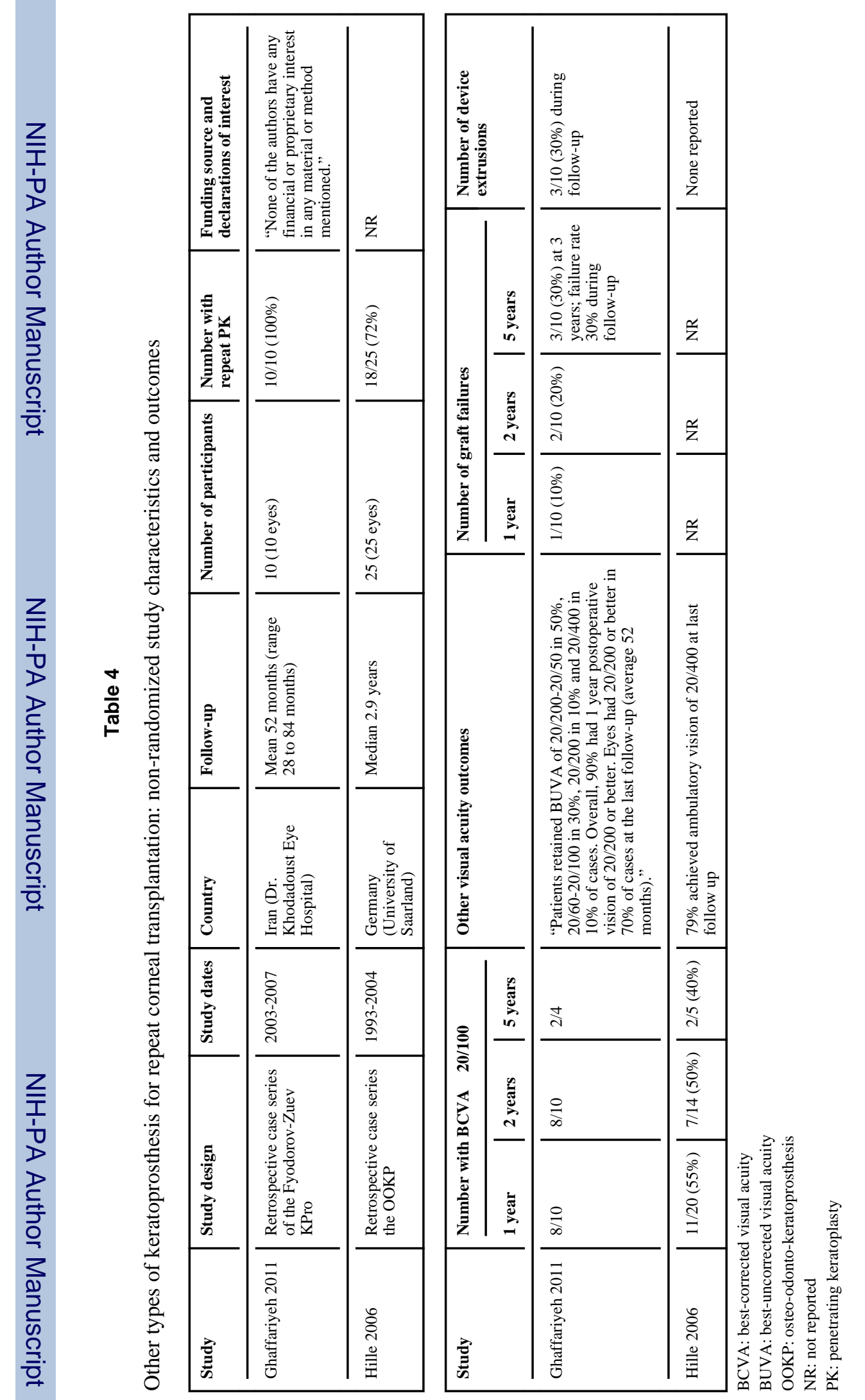

Cochrane Database Syst Rev. Author manuscript; available in PMC 2014 December 18. 
Characteristics of excluded studies [ordered by study ID]

\begin{tabular}{|l|l|}
\hline Study & Reason for exclusion \\
\hline Aldave 2012a & Retrospective case series of Boston keratoprosthesis \\
\hline Aldave 2012b & $\begin{array}{l}\text { Retrospective case series of Boston keratoprosthesis;international cohort compared with cohort from Aldave } \\
\text { 2012a }\end{array}$ \\
\hline Aquavella 2005 & Retrospective case series of Boston keratoprosthesis (Dohlman-Doane model) \\
\hline Boston Type 1 KPro Study & Multicenter prospective and retrospective case series of Boston keratoprosthesis \\
\hline Chew 2009 & Retrospective case series of Boston keratoprosthesis \\
\hline Dunlap 2010 & Retrospective case series of Boston keratoprosthesis \\
\hline Ghaffariyeh 2011 & Retrospective case series of Fyodorov-Zuev keratoprosthesis \\
\hline Greiner 2011 & Retrospective case series of Boston keratoprosthesis \\
\hline Guell 2011 & Retrospective case series of Boston keratoprosthesis \\
\hline Harissi-Dagher 2007 & Retrospective case series of Boston keratoprosthesis (Dohlman-Doane model) \\
\hline Hicks 2006 & Multicenter prospective and retrospective surveillance study of AlphaCor \\
\hline Hille 2006 & Retrospective case series of osteo-odonto-keratoprosthesis \\
\hline Jiraskova 2011 & Retrospective case series of AlphaCor \\
\hline Kamyar 2012 & Retrospective case series of Boston keratoprosthesis \\
\hline Koller 2012 & Retrospective case series of Boston keratoprosthesis \\
\hline Patel 2012 & Retrospective case series of Boston keratoprosthesis \\
\hline Shihadeh 2012 & Retrospective case series of Boston keratoprosthesis \\
\hline Talajic 2012 & Retrospective case series of Boston keratoprosthesis \\
\hline Trichet 2013 & Retrospective case series of AlphaCor \\
\hline
\end{tabular}

